\title{
A PROdUÇÃO CIENTÍFICA ACERCA DA COMUNICAÇÃO EM ENFERMAGEM
}

\author{
Hujiko Enoki* \\ Ana Emilia Pace Ferraz* \\ Emilia Campos de Carvalho* \\ Maria Helena Palucci Marziale*
}

\begin{abstract}
RESUMO - Teve-se por objetivo, conhecer a produção científica acerca de comunicação em Enfermagem em cinco periódicos nacionais, a partir de 1970. Os artigos foram classificados por área de predomínio, tipo metodológico utilizado, função do autor principal e ano de publicação. Os resultados evidenciaram serem os artigos em sua maioria de docentes, com metodologia básica exploratória, de reflexão teórica e quantitativa descritiva; quanto à área, houve predomínio de artigos na assistencial, seguidos da administrativa e por fim a área educativa.
\end{abstract}

ABSTRACT - The objective of the presente study was to survey the scientific production concerning communication in Nursing in five Brazilian journals, starting from 1970. Articles were classified by area of predominance, type of methodology used, function of the main author, and year of publication. The results showed that most articles were written by university teachers, using basic exploratory, theoretical reflection, and quantitative descriptive methodologies. There was a predominance of articles concerning assistance, followed by article in the administrative area and in the educational area.

\section{INTRODUÇÃO}

0 interesse em conhecer a atual fase de produção científica de comunicação em enfermagem dentro do contexto geral da pesquisa em enfermagem, no Brasil, motivou a elaboração do presente trabalho. A produção do conhecimento em Enfermagem já foi objeto de vários estudos como os de VIEIRA (1980), ALMEIDA (1981), NOGUEIRA (1982) e NEVES (1982).

A enfermagem como vocação, arte técnica ou profissão, em qualquer que seja o contexto sócio-cultural considerado, depende sempre da comunicação entre seres humanos. 0 enfermeiro poderá, como comunicador em potencial, interferir em uma dada situação, tornando-a efetiva através do conhecimento dos elementos, que atuam como adjuvantes ou oponentes ao processo de comunicação.

A comunicação é considerada como instrumento básico na Enfermagem conformef risam HORTA (1970), KAMYAMA \& PAULA (1970). Estas autoras transmitem a idéia de que, os instrumentos representam o conjunto de conhecimentos e habilidades fundamentais para o exercício de todas as atividades profissionais e distinguem a comunicação como essência da interação entre enfermeiro-paciente.

0 reconhecimento da importância sobre comunicação em enfermagem, possibilitou que se constituisse um dos temas oficiais do XXI Congresso Brasileiro de Enfermagem (1969). Este fato constitui-se marco para o início do nosso levantamento, cujo objetivo principal foi reconhecer a produção científica acerca da Comunicação em Enfermagem em cinco (05) períodicos nacionais.

\section{METODOLOGIA DO ESTUDO}

0 primeiro passo constitui-se da definição e delimitação da abrangência da produção científica a respeito de Comunicação em Enfermagem no Brasil. Foram portanto, consultadas dentre as principais fontes de referências de pesquisa em enfermagem, os periódicos:

\footnotetext{
* Docente do Departamento de Enfermagem Geral e Especializada da Escola de Enfermagem de Ribeirão Preto - USP.
} 
- Revista Brasileira de Enfermagem (1970 - 1985)

- Revista Paulista de Enfermagem (1981 - 1985)

- Enfermagem Atual (1987 - 1982)

- Enfermagem Moderna (1983 - 1985)

- Enfermagem em Novas Dimensões (1975 - 1979)

Os critérios para seleção dos trabalhos a serem analisados foram: terem sido publicados nos periódicos citados a partir de 1970 e terem sido realizados por enfermeiros.

Os dados levantados foram classificados de acordo com os critérios:

- Ano de publicação e periódico

- Área de atuação do autor principal mencionada na publicação: docência, assistencial, docenteassistencial, administração em geral, outros e não especificado.

- Área de predomínio do trabalho: educação, administração e assistência.

- Metodologia básica do trabalho: exploratório, experimental, descritivo quantitativo, descritivo de caso, reflexão teórica e relato de experiência na prática de enfermagem.

\section{RESULTADOS E COMENTÁRIOS}

Dos periódicos selecionados para análise, foram identificados 33 artigos a partir de 1970 .

Como se pode verificar na Tabela 1 , apesar do reconhecimento da relevância do tema em 1969 no XXl Congresso Brasileiro de Enfermagem, não se observou imediata repercussão na produção de trabalhos nesta área de conhecimento, visto que nos anos de 1970 1973 , não se identificou publicação sobre comunicação. Nos anos subseqüentes, existem variações de um a quatro artigos por ano e o maior índice apresentado foi no ano de 1976 com oito publicações.

Do total de publicações (Tabela 1), a Revista Brasileira de Enfermagem é a que tem maior número, totalizando 54,55\%, talvez por ser ela segundo KOIZUMI et alii (1985), a publicação de maior tradição e penetração em todo o país, por contar com a colaboração de autores das diferentes regiões brasileiras e por abranger todas as áreas de connecimento da enfermagem. Em seguida, os perioódicos: Enfermagem em Novas Dimensões (18,18\%), Revista Paulista de Hospitais,

TABELA 1 - Freqüência de artigos publicados sobre comunicação em enfermagem, no período de 1970 a 1985 segundo periódico e área predomínio do trabalho.

\begin{tabular}{|c|c|c|c|c|c|c|c|c|c|c|c|}
\hline \multirow[b]{2}{*}{ ANO } & \multicolumn{5}{|c|}{ PERIÓDICOS } & \multicolumn{5}{|c|}{ ÁREA DE PREDOMÍNIO DO TRABALHO } & \multirow[b]{2}{*}{ TOTAL } \\
\hline & REBEn & $\begin{array}{c}\text { ENF. } \\
\text { NOVAS } \\
\text { DIMENSŌES }\end{array}$ & $\begin{array}{l}\text { REV. } \\
\text { PAUL. } \\
\text { HOSP. }\end{array}$ & $\begin{array}{l}\text { ENF. } \\
\text { ATUAL }\end{array}$ & $\begin{array}{c}\text { ENF. } \\
\text { MODERNA }\end{array}$ & $\begin{array}{r}\text { EDUCAÇ } \\
\text { EDUCAÇÃO }\end{array}$ & $\begin{array}{l}\text { ÇÀO } \\
\text { EQUIPE }\end{array}$ & $\begin{array}{r}\text { AS } \\
\text { HOSP. }\end{array}$ & $\begin{array}{l}\text { ISTENCIAL } \\
\text { AMB. COMUN. }\end{array}$ & ADMINISTRAÇĀ0 & \\
\hline $1970-73$ & & & & & & & & & & & \\
\hline 1974 & 3 & & & & & 1 & & 1 & & 1 & 3 \\
\hline 1975 & 2 & 2 & & & & 1 & & 3 & & & 4 \\
\hline 1976 & 7 & 1 & & & & & 1 & 3 & & & 8 \\
\hline 1977 & 2 & 1 & & & & & & 2 & & 1 & 3 \\
\hline 1978 & 0 & 2 & & & & & & 2 & & & 2 \\
\hline 1979 & 1 & & & & & & & 1 & & & 1 \\
\hline 1980 & 1 & & & 2 & & & & 1 & & 2 & 3 \\
\hline 1981 & & & & 2 & & & & 1 & & 1 & 2 \\
\hline 1982 & & & 1 & & & & & & & 1 & 1 \\
\hline 1983 & & & & & 1 & & & 1 & & & 1 \\
\hline 1984 & 1 & & 1 & & & & & 1 & 1 & & 2 \\
\hline 1985 & 1 & & 2 & & & & & 2 & & 1 & 3 \\
\hline $\begin{array}{l}\text { FREQÜEN. } \\
\text { SIMPLES }\end{array}$ & 18 & 6 & 4 & 4 & 1 & 1 & 1 & 18 & 1 & 11 & 33 \\
\hline $\begin{array}{l}\text { FREQÜEN. } \\
\text { PERCENTUAL }\end{array}$ & 54,55 & 18,18 & 12,12 & 12,12 & 3,03 & 9,09 & & & 57,58 & 33,33 & $100 \%$ \\
\hline
\end{tabular}

Enfermagem Atual com $(12,12 \%)$ e Enfermagem Moderna (3,03\%).

Em relação a área de predomínio dos trabalhos sobre comunicação em Enfermagem, encontrou-se que a assistencial foi a mais freqüente $(57,58 \%)$, seguida da administrativa (33,33\%) e de educação (9,09\%). Estes resultados parecem concordar com o verificado por
NEVES (1982), pois para esta autora as pesquisas em enfermagem relacionam-se mais freqüentemente com a área assistencial. Também segundo ALMEIDA et alii (1981), através de um estudo realizado para avaliar a produção de dissertações e teses de enfermagem, as pesquisas nesta área se concentram primordialmente na busca de melhoria da assistência, isto é, nos aspec- 
tos internos da prática, enquanto prática técnica. Outra autora que apresenta resultados semelhantes é NOGUEIRA (1982), que analisando pesquisas de enfermagem no Brasil também constata que as linhas principais de pesquisa, desde 1950 até 1981 , relacionam-se, principalmente, aos aspectos metodológicos da assistência, seguido dos relativos ao ensino da enfermagem. Segundo esta autora, estas linhas parecem estar correspondendo prioritariamente às necessidades do momento histórico ou sendo influenciadas pelas práticas de saúde decorrentes da evolução social do país.

A segunda área de predomínio dos trabalhos mais abortada pelos autores, a respeito de comunicação, foi a administrativa (33,33\%). Evidenciou-se preocupação acentuada (8 entre 11 artigos) com a comunicação escrita, tais como anotação em prontuários e elaboração de instrumentos de registro de atividades.

$\mathrm{Na}$ área de educação $(9,09 \%)$ foram considerados os artigos relativos a educação de pessoal e de alunos de enfermagem.

Ressaltam-se que, foi observada uma freqüente associação entre comunicação e orientação do paciente. Os artigos que só continham a comunicação implícita, sem ser o tema abordado no trabalho, não foram incluídos neste estudo e aqueles que explicitavam a comunicação no processo de orientação do paciente, foram computados na área assistencial.

Quanto à área de atuação do autor principalmente dos 33 artigos, $(75,76 \%)$ foram ef etuados por docen- tes, $(21,21 \%)$ por enfermeiros de serviço e $(3,03 \%)$ por outros. Segundo MENDES \& TREVISAN (1983), o despreparo do enfermeiro com relação a elaboração de pesquisas e a aplicação do produto das pesquisas à prática, a pequena ênfase que tem sido dada nos currículos dos cursos de graduação de enfermagem à metodologia da pesquisa e a falta de cursos de especialização sobre metodologia de pesquisa para enfermeiros, estejam contribuindo para o baixo índice de trabalhos efetuados por enfermeiros de serviço.

Abordando ainda a formação dos autores, LEMOS (1985) ressalta que a produção científica tomou impulso após a instalação de cursos de pós-graduação na década de 70, que com a aquisição do conhecimento dos processo metodológicos, levaram os profissionais a se iniciarem na pesquisa, o que pode justificar os $75,76 \%$ do total de artigos publicados por docentes.

Quanto a metodologia básica em que os trabalhos publicados foram elaborados, observa-se uma predominância do tipo exploratório (30,30\%), de reflexão teórica $(27,27 \%)$ seguidos pelos descritos quantitativos $(21,21 \%)$, descritivo de caso $(12.12 \%)$ e relato de experiência $(6,06 \%)$; não se encontrou o tipo trabalho experimental. Um dos trabalhos não foi enquadrado nos tipos metodológicos por se tratar de uma reportagem (3,03\%).

Para visualização das diferentes metodologias utilizadas no período observado foi elaborado o Gráfico 1.

GRÁFICO 1 Distribuição das metodologias básicas dos artigos sobre comunicação no período de 1970 a 1985.

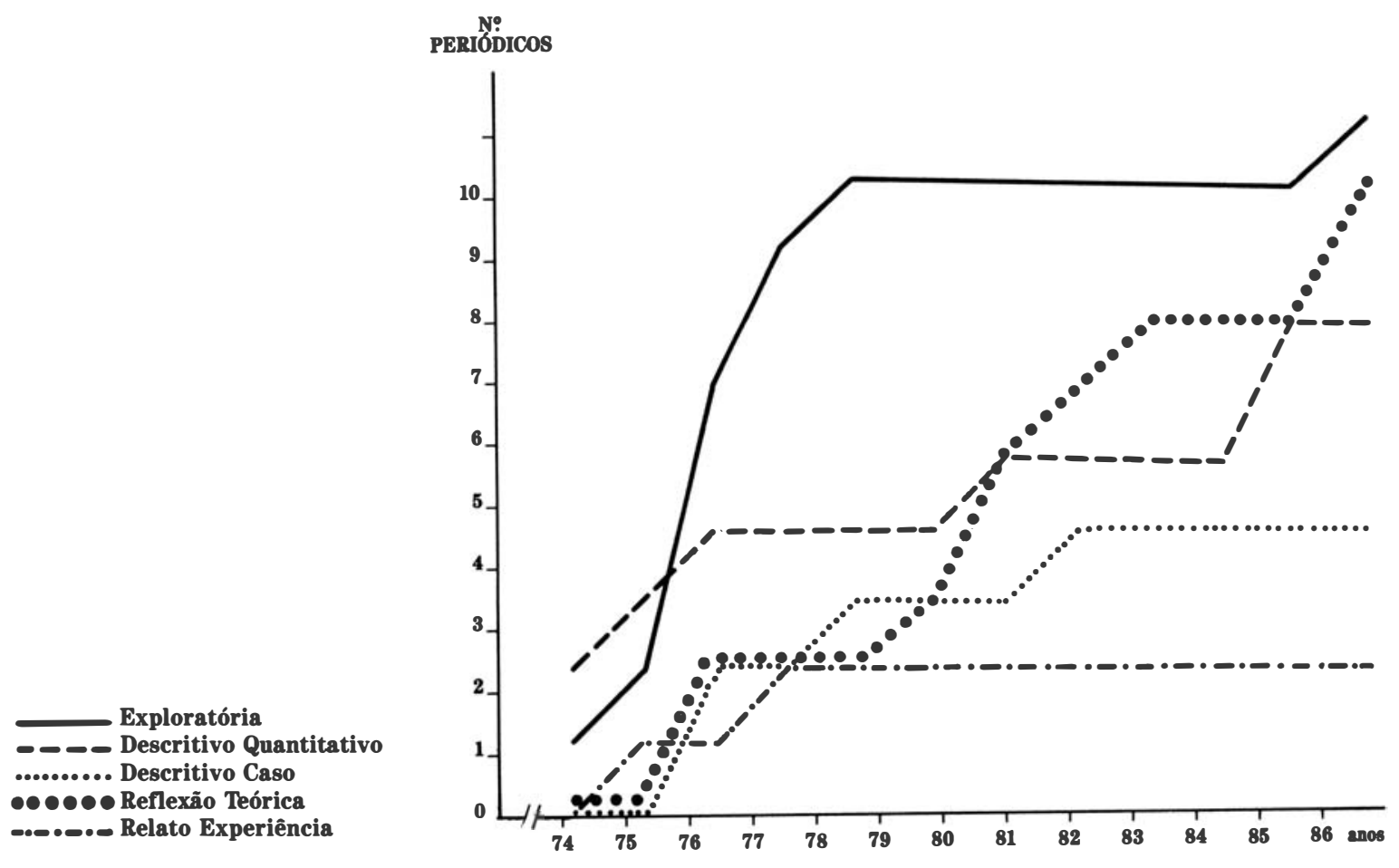


Ressalta-se novamente que, no período de 1970 a 1973 não houve publicações acerca deste tema, respeitados os critérios adotados no presente trabalho.

Buscando uma distribuição da freqüência de produção científica durante o período observado, nota-se que em 1976, prevaleceram trabalhos do tipo exploratório, onde a ênfase principal está na articulação de conceitos, na formulação de um problema e desenvolvimento de hipóteses a serem testadas. Outros dois tipos metodológicos que se sobressairam neste mesmo ano foram de reflexão teórica e descritivo de caso; 0 primeiro constituiu-se de uma exposição lógica e reflexiva de um tema e uma argumentação com interpretação e julgamento pessoal, enquanto que o segundo, há descrição de uma situação levando em conta 0 estudo de grande número de variáveis em um caso ou um número relativamente pequeno de casos.

Em 1980 e 1985 o predomínio foi do tipo reflexão teórica e em 1984 do quantitativo descritivo, o qual descreve uma situação levando em conta o estudo de poucas variáveis em grande número de casos.

Outro tipo metodológico encontrado, em menor evidência foi o relato de experiência, a qual descreve, e comenta uma situação vivenciada na prática. Foi ainda incluída uma publicação de entrevista realizada na área deste estudo, mais precisamente sobre o diálogo enfermeiro-paciente.

De maneira geral, pode-se observar que houve 0 emprego de diferentes tipo metodológicos, utilizandoos em sua maior ou menor abrangência, em trabalhos de maior ou menor complexidade. Tem-se a ressaltar que, a maior utilização de trabalhos exploratórios, descritivo quantitativos e de reflexão teórica refletem a incipiência do conhecimento na área de comunicação. Esses achados parecem concordar com o de NEVES (1982) e MENDES \& TREVISAN (1983) em trabalhos acerca da utilização de metodologias de pesquisa em Enfermagem.

Tem-se que ressaltar na consideração destes dados, a abrangência do presente trabalho, que não inclui as recentes teses e monografias desenvolvidas sobre 0 assunto.

\section{CONSIDERAÇÕES FINAIS}

Através da quantificação e classificação dos artigos sobre comunicação, pode-se detectar que:

- A produção científica acerca da Comunicação em Enfermagem, nos periódicos citados, nos últimos 15 anos, perfez um total de 33 publicações, com maior freqüência no período de 1974 a 1977, com 54,54\% dos artigos, sendo que se destaca o ano de 1976, quando se observou o maior índice de publicação/ano com 8 artigos.

- A área de aplicação dos trabalhos mais freqüente foi a assistencial (57,58\%), seguida da administrativa $(33,33 \%)$ e de educação $(9,09 \%)$.

- Os autores principais são em sua maioria docentes $(75,76 \%)$, seguidos de autores com atividade assistencial $(21,21 \%)$ e outros $(3,03 \%)$.

- As metodologias básicas mais freqüentes foram: exploratórias $(30,30 \%)$, reflexão teórica $(27,27 \%)$, quantitativo descritiva $(21,21 \%)$, descritivo de caso $(12,12 \%)$ e relato de experiência $(6,06 \%)$ : salienta-se que, 1(um) não se enquadra por ser reportagem.

Os dados evidenciaram ainda, que a comunicação como essência da interação enfermeiro-paciente como frisam HORTA (1970), KAMIYAMA \& PAULA (1970), foi abordada em $15,15 \%$ dos artigos analisados, o que sugere maior necessidade de estudo nesta área de comunicação.

Outro aspecto observado refere-se à terminologia divergente utilizada pelos autores, sugerindo também ser este um outro campo de pesquisas em Enfermagem.

\section{REFERÊNCIAS BIBLIOGRÁFICAS}

1. LEMOS, M. J. A. - Conhecimento que o enfermeiro deve possuir para se iniciar como pesquisador. In: Seminário de Pesquisa em Enfermagem, 4. São Paulo, 1985. Anais do 40 Seminário de Pesquisa em Enfermagem p. 118-26.

2. MENDES, I.A.C. \& TREVISAN, M.A. - A cerca da utilização do método científico nas pesquisas de enfermagem. Revista Brasileira de Enfermagem. Brasília 36(1):13-9, jan./fev./mar. 1983.

3. ALMEIDA, M.C.P. et alii. A produção do conhecimento na pósgraduação em Enfermagem no Brasil. In: CONGRESSO BRASILEIRO DE ENFERMAGEM, 33. Manaus, 2-7 agosto, 1981. Anais do 4 ? Seminário de Pesquisa em Enfermagem p. 119-26.

4. HORTA, W. de A. Dos instrumentos básicos de enfermagem. Revista da Escola de Enfermagem da USP. São Paulo, 4 (1/2): 2-4. Mar./Set. 1970.

5. NEVES, E.P. - Vazios do conhecimento e sugestões de temáticas relevante na área de Enfermagem. In: SEMINÁRIO NACIONAL SOBRE ENSINO DA PÓS-GRADUAÇÃO E PESQUISA EM ENFERMAGEM, 2, Brasília, 1982. Anais do $4^{\circ}$. Seminário de Pesquisa em Enfermagem, p.20-50.

6. NOGUEIRA, M. J. de C. - A pesquisa em Enfermagem no Brasil: Retrospectiva Histórica. Revista da Escola de Enfermagem da USP. São Paulo 16(1): 17-26, abr. 1982.

7. KOIZUMI, M. S. et alii - Pesquisa em Enfermagem Médico Cirúrgica. In: SEMINÁRIO NACIONAL DE PESQUISA EM ENFERMAGEM, São Paulo, 1985. Anais do 4\% Seminário de Pesquisas em Enfermagem, p. 60-77.

7. VIEIRA, T.V. - Produção científica em Enfermagem no Brasil: 1960-1970. Salvador-Bahia, UFBA, 1980 (Tese Prof. Titular). 